\title{
Política y cultura en don Juan Manuel (1320-1325) ¿Un proyecto de exaltación neoalfonsí?
}

\author{
Politics and Culture in don Juan Manuel (1320-1325) \\ A neoalphonsine exaltation project? \\ JOSÉ ÁNGEL SALGADO LOUREIRO \\ Universidad de Santiago de Compostela \\ asalgado.loureiro@gmail.com \\ https://orcid.org/0000-0003-1526-4925 \\ http://dx.doi.org/10.15304/sm.31.5999
}

\section{RESUMEN}

Las obras escritas por don Juan Manuel entre 1302 y 1325 se caracterizan en conjunto por presentar paralelismos con la producción narrativa de Alfonso X. Bajo la idea de un "proyecto neoalfonsí", estudiaremos la posibilidad de que estas obras de don Juan Manuel formasen parte de un programa político con fines específicos, localizado en el marco de las luchas por la tutoría durante la minoría de edad de Alfonso XI. Para ello, estudiaremos los contextos político-biográfico e ideológicocultural del autor, observando como ambos se relacionan al interpretar la emulación que realiza del rey Sabio.

Palabras clave: don Juan Manuel, Alfonso X, Fernando IV, molinismo, Corona de Castilla.

\begin{abstract}
The works written by Don Juan Manuel between 1302 and 1325 are characterized together by presenting parallelisms with the narrative production of Alfonso X. On the basis of the idea of a "neoalphonsin proyect", we will study the possibility that these works of Don Juan Manuel had been part of a political program with specific purposes, located within the framework of the confrontations for tutoring during the minority of Alfonso XI. In order to achieve this, we will study the author's political-biographical and ideological-cultural context, noticing how both relate when we interpret the emulation performed of the Wise King.
\end{abstract}

Keywords: don Juan Manuel, Alfonso X, Fernando IV, molinismo, Crown of Castile.

\section{INTRODUCCIÓN}

En los estudios sobre el panorama político y cultural de la Corona de Castilla, don Juan Manuel ha destacado como una de las figuras más relevantes entre finales del siglo XIII y la primera mitad del siglo XIV. Como miembro de la familia real castellana, 
destacó por una alta consideración de su persona en un plano linajístico que lo llevó a participar de forma activa en los enfrentamientos nobiliarios que se sucedieron entre los reinados de Fernando IV y Alfonso XI. ${ }^{1}$ En lo que se refiere a su faceta cultural, su producción narrativa ha constituido un elemento de obligada referencia en los estudios sobre la historia de la literatura y las representaciones sociopolíticas medievales castellanas. $^{2}$

Un tema clásico en el estudio de la obra de don Juan Manuel ha sido su vinculación con la figura autoral de Alfonso X a través, especialmente, de la primera mitad de su producción, que comprende desde 1320 a $1325 .{ }^{3}$ Esto ha contrastado con algunas investigaciones recientes que lo han vinculado con el posterior entorno cultural de los reinados de Sancho IV y Fernando IV, caracterizado por su reacción al proyecto político y cultural alfonsí. A raíz de ello se ha establecido que el vínculo de don Juan Manuel con Alfonso $\mathrm{X}$ es meramente cultural y no político. ${ }^{4}$

La hipótesis que aquí planteamos es la de que el bloque de obras de don Juan Manuel compuestas entre 1320 y 1325, sobre las que se ha establecido su relación con Alfonso $\mathrm{X}$, puedan haber constituido un proyecto político del autor a través de la valorización de la imagen del gobernante intelectual. Este proyecto habría que entenderlo como consecuencia, al mismo tiempo, de su experiencia política y de su bagaje ideológico y cultural. Por ello, estructuraremos este trabajo en tres puntos. En el primero, "La autoconciencia nobiliar de don Juan Manuel como miembro de la familia real", estudiaremos cómo el contexto histórico y biográfico del autor sirvieron para moldear sus planteamientos políticos, así como la imagen que tenía de sí mismo. A partir de ahí podremos tratar en el segundo punto, "El gusto de don Juan Manuel por la cultura en el marco ideológico del molinismo", cómo don Juan Manuel se inserta en el marco ideológico de su época y la forma en la que, sobre éste, desarrolla un gusto particular por las letras y la cultura. Hecho esto podremos abordar en el tercer punto, "La recepción de Alfonso X por don Juan Manuel desde el marco ideológico del molinismo", la forma en que recibe la herencia

1 Una obra básica para el estudio de la trayectoria política de don Juan Manuel es el ya clásico estudio biográfico de Giménez Soler: Giménez Soler, A., Don Juan Manuel. Biografía y estudio crítico, Zaragoza, 1932. También son frecuentes las referencias a su figura en estudios monográficos dedicados la historia política del momento, como los de González Míguez sobre Fernando IV, y Sánchez-Arcilla sobre Alfonso XI: González Mínguez, C., Fernando IV (1295-1312), Palencia, 1995; Sánchez-Arcilla Bernal, J., Alfonso XI 1312-1350, Palencia, 1995.

2 Gómez Redondo dedicó a don Juan Manuel una amplia sección en su monumental Historia de la prosa medieval castellana: Gómez Redondo, F., Historia de la Prosa Medieval Castellana, I, Madrid, 1998, pp. 1103-1202. También ha sido objeto de especial interés por parte de María Jesús Lacarra, quien le dedicó une estudio monográfico en 2006: Lacarra, M. J., Don Juan Manuel, Madrid, 2006.

3 Orduna, G., "Los prólogos a la Crónica Abreviada y al Libro de la Caza: la tradición alfonsí y la primera época en la obra literaria de don Juan Manuel", Cuadernos de Historia de España, 51-52, 1970, pp. 123-144.

4 Gómez Redondo, F., "Don Juan Manuel, autor molinista”, en Margarita Freixas, Silvia Iriso Ariz y Laura Fernández (coords.), Actas del VIII Congreso Internacional de la Asociación Hispánica de Literatura Medieval, I, Santander: Asociación Hispánica de Cultura Medieval, 2000, pp. 827-842. 
cultural de Alfonso X. Una vez vistas estas tres cuestiones, estaremos en posición de concluir cómo don Juan Manuel utiliza esta herencia cultural con una finalidad política dentro de su contexto.

\section{LA AUTOCONCIENCIA NOBILIAR DE DON JUAN MANUEL COMO MIEMBRO DE LA FAMILIA REAL}

Para comprender la forma en que don Juan Manuel construye su autoconciencia nobiliaria a través de la experiencia hemos considerado oportuno dividir este apartado en tres partes. Empezaremos presentando el origen familiar de don Juan Manuel como ricohombre castellano y miembro de la familia real. En segundo lugar, observaremos la forma en que se insertó en los conflictos nobiliarios durante el reinado de Fernando IV. Finalmente trataremos la situación de don Juan Manuel durante la minoría de edad de Alfonso XI (1312-1325), periodo dentro del cual inicia su labor de escritura.

\subsection{El origen familiar: hijo del infante don Manuel y adelantado del Reino de Murcia}

Para comprender la posición social desde la que escribe don Juan Manuel hay que partir de su origen familiar. Nació el 5 de mayo de 1282 en Escalona, fruto del matrimonio entre el infante don Manuel con su segunda esposa, doña Beatriz de Saboya. Por parte de padre se vincula con la familia real castellana siendo nieto del rey Fernando III el Santo, sobrino del rey Alfonso X el Sabio y primo del rey Sancho IV el Bravo. Por parte de madre es nieto del conde Amadeo IV de Saboya, lo que lo emparentaba con la nobleza del Imperio Romano Germánico. ${ }^{5}$ Se sitúa así en lo que parece haber sido, en cuanto a parentesco, el escalón más bajo de la familia real. Ahora bien, la pertenencia al linaje regio constituyó una de sus principales señas de identidad, tal y como atestigua su intitulación tanto en la documentación oficial y epistolar de la época, así como en los prólogos de sus obras. En todas las ocasiones se lo menciona como don Juan, hijo del infante don Manuel. ${ }^{6}$

En lo que al patrimonio se refiere, obtuvo un extenso señorío por herencia paterna tras la muerte del infante don Manuel en 1283. Destacaron en sus posesiones la tenencia de señoríos como el de Peñafiel, así como el título de adelantado del Reino de Murcia,

5 Sobre esto véase: Giménez Soler, A., Don Juan Manuel, op. cit., pp. 1-2; Lacarra, M. J., Don Juan Manuel, op. cit., pp. 9-12; Flory, D., El Conde Lucanor: don Juan Manuel en su contexto histórico, Madrid: Pliegos, 1995, p.13.

6 A este efecto, Derek Lomax se aproximó a la influencia que habría tenido sobre don Juan Manuel la figura de su padre. Véase: Lomax, D., "El padre de don Juan Manuel”, Don Juan Manuel: VII Centenario, Murcia, 1982, pp. 163-176. 
que ya había ostentado su padre, y que el rey Sancho IV le conservó de forma nominal. ${ }^{7}$ Siendo apenas un niño, el cargo de adelantado lo situaba entre uno de los principales oficiales de la administración regia. De él dependían las funciones militares, fiscales y jurídicas sobre Murcia. De hecho, la importancia del cargo hacía que con frecuencia recayese en miembros de la familia real. ${ }^{8}$

Varios documentos entre 1284 y 1295 informan sobre una participación temprana, aunque progresiva, de don Juan Manuel en las funciones gubernativas del adelantamiento de Murcia junto con sus lugartenientes. Podemos considerar que también se inició con precocidad en las funciones militares puesto que, en 1294, a la edad de doce años, estuvo presente en los preparativos para la defensa de Murcia frente a Granada, no llegando a entrar en combate debido a su juventud. ${ }^{9}$

Podemos apreciar así que la autoconciencia nobiliar de don Juan Manuel puede resultar un tanto compleja, situándose entre dos grupos sociales, como son la familia real y la alta nobleza. En esto, parece encajar con la ambigüedad de su posición social como hijo de infante. En la Castilla del siglo XIV la distinción de estatus entre la nobleza no tiene una base jurídica clara, sino que es más bien subjetiva. De forma general, un hijo de infante tendía a ser ubicado más bien dentro de la alta nobleza. ${ }^{10}$ De hecho, el comportamiento político de don Juan Manuel fue, en general, el propio de un miembro de este grupo. Esto se puede apreciar en la actitud que mantuvo hacia el adelantamiento de Murcia. El hecho de haberlo recibido como herencia y la ausencia de la autoridad regia tras la muerte de Sancho IV, condicionaron la formación de un sentimiento de propiedad patrimonial sobre el territorio. Por ello, su gobierno se caracterizó como un régimen de tipo señorial, muy alejado de las funciones reales que le atañían como oficial de la Corona. ${ }^{11}$ Sin embargo, la cuestión del vínculo con el círculo familiar regio en tanto que hijo de infante parece haber tenido especial peso en la comprensión personal sobre su estatus social.

7 Sobre esto véase: Rubio García, L., La minoridad de don Juan Manuel y la ocupación aragonesa de Murcia (1282-1296), Murcia: Real Academia Alfonso X el Sabio, 2000, pp. 16-30; Giménez Soler, A., Don Juan Manuel, op. cit., p. 3.

8 Ostolaza Elizondo, M. I., "La Cancillería y otros organismos de expedición de documentos durante el reinado de Alfonso XI (1312-1350)", Anuario De Estudios Medievales, 16, 1986, pp. 158-159.

9 Sobre esto véase: Rubio García, L., La minoridad, op. cit., pp. 16-30, pp. 71-81 y pp. 144-145; Giménez Soler, A., Don Juan Manuel, op. cit., pp. 3-9; Torres Fontes, J., "Murcia y don Juan Manuel: tensiones y conflictos", en Don Juan Manuel: VII centenario, Murcia, 1982, pp. 359-360.

10 Para esta afirmación nos basamos en el estudio de Salvador Moxó: Moxó, S., "La nobleza castellana en el siglo XIV", (coords.) Pablo Sánchez León y Jesús Izquierdo, Clásicos de Historia Social de España: Una Selección Crítica, Valencia, Centro Francisco Tomás y Valiente (UNED), 2000, p. 96. Sus planteamientos se centran en el inicio de los cuadernos de Cortes de la época, donde se alude a los grupos sociales presentes en cada celebración. Ahora bien, cabe notar que este planteamiento estrictamente jurídico de Moxó debería de ser contrastado con las pocas evidencias de que disponemos (y don Juan Manuel es una de ellas) sobre la forma subjetiva en que la nobleza se veía a sí misma.

11 Sobre esto véase: Moreno Núñez, J. I., "Algunas consideraciones y documentos sobre el régimen señorial en el tránsito de la Baja Edad Media”, Anuario De Estudios Medievales, 16, 1986, pp. 115-117; Torres Fontes, J., "Murcia y don Juan Manuel”, op. cit., p. 356. 


\subsection{Privanza y rebeldía durante el reinado de Fernando IV}

En los principales estudios sobre el reinado de Fernando IV se ha tendido a dividirlo en dos etapas atendiendo al desarrollo de diferentes tipos de conflicto interno. La primera, que ocupó su minoría de edad y el inicio de su gobierno hasta 1304, estuvo condicionada por la rebelión del infante de la Cerda. La segunda, desde 1305 hasta la muerte del Rey en 1312, se caracterizó por los enfrentamientos entre bandos nobiliarios en pugna por la consecución de intereses particulares. ${ }^{12}$

En la primera etapa, el infante don Alfonso de la Cerda se rebeló junto con el infante don Juan, siendo ambos, respectivamente, nieto e hijo de Alfonso X el Sabio. ${ }^{13}$ Una parte considerable de la nobleza castellana se agrupó en bandos en torno a los dos rebeldes, que contaron también con el apoyo de las coronas de Aragón y Portugal. ${ }^{14}$ Por su parte, don Juan Manuel mantuvo las lealtades de su padre, que había apoyado a Sancho IV, por lo que permaneció al lado de Fernando IV y María de Molina. A razón de ello tuvo que hacer frente a las agresiones de Jaime II de Aragón, que buscaba apoderarse de su adelantamiento del Reino de Murcia. ${ }^{15}$

La solución a este enfrentamiento, que había alcanzado dimensiones de auténtica guerra civil, llegó entre las Cortes de Valladolid de 1300 y el Tratado de Elche en 1304. El resultado pudo haber sido en cierto modo insatisfactorio para don Juan Manuel. Mientras él se veía obligado a renunciar a una porción considerable de sus posesiones en Murcia, los rebeldes que volvían al servicio de Fernando IV no sólo no recibían penalizaciones, sino que obtenían beneficios por su rendición. ${ }^{16}$ A partir de esto podría haber

12 Tal división fue planteada por González Mínguez en 1976 y mantenida, con algunos matices, en sus trabajos posteriores. Véanse: González Mínguez, C., "Fernando IV de Castilla (1295-1312): La guerra civil y el predominio de la nobleza", Valladolid, 1976, pp. 327-328; González Mínguez, C., "Fernando IV de Castilla (1295-1312) perfil de un reinado", Espacio, tiempo y forma, 17, 2004, p. 235

13 Sobre esto véase: Masià de Ros, A., "Las pretensiones de los infantes de la Cerda a la Corona de Castilla en tiempos de Sancho IV y Fernando IV: el apoyo aragonés”, Medievalia, 10, 1992, pp. 255-257; González Mínguez, C., Fernando IV (1295-1312), op. cit., pp. 21-93. De igual modo, sobre los conflictos a finales del reinado de Alfonso X puede verse: Rodríguez Llopis, M., “Alfonso X, rey de Castilla y León (1252-1284)", en Rodríguez Llopis, M. (coord.), Alfonso X y su época: el siglo del rey sabio, Barcelona, 2001, pp. 107-125.

14 Sobre esto véase: González Mínguez, C., "Fernando IV de Castilla (1295-1312) perfil de un reinado", pp. 226-241; González Mínguez, C., Fernando IV (1295-1312), op. cit., pp. 65-66; Nussbaum, M. F., "La imagen jurídica del rey en la Crónica de Fernando IV", en Martínez Pérez, A. y Baquero Escudero, A. L. (coords.), Estudios de literatura medieval: 25 años de la Asociación Hispánica de Literatura Medieval: 25 años de la AHLM, Murica, 2012, p. 645.

15 Sobre esto véase: Giménez Soler, A., Don Juan Manuel, op. cit., pp. 6-9; González Mínguez, C., Fernando IV (1295-1312), op. cit., pp. 74-82, pp. 31-41; Masià de Ros, A., "Las pretensiones", op. cit., pp. 257-259.

16 Sobre esto véase: López Serrano, A., Jaime II, don Juan Manuel y el Señorío de Villena, Villena, 1999 , pp. 35-43; O'Callaghan, J. F., "Las Cortes de Fernando IV: cuadernos inéditos de Valladolid 1300 y Burgos 1308", Historia. Instituciones. Documentos, 13, 1986, p. 317; González Mínguez, C., "Fernando IV de Castilla (1295-1312) perfil de un reinado", pp. 228-230; González Mínguez, C., Fernando IV (1295-1312), op. cit., pp. 65-74; Masià de Ros, A., "Las pretensiones", op. cit., p. 261. 
comprendido que la consecución del beneficio personal habría de lograrse a partir de la habilidad y la actuación individual, y no estrictamente de la obediencia al Rey. De este modo, durante la segunda etapa del reinado de Fernando IV se sumó a las filas de los nobles levantiscos, actuando como uno de los principales apoyos del infante don Juan.

Durante la segunda etapa, podemos comprender que la conflictividad política y militar se debería a las tensiones entre miembros de la alta nobleza, y no al enfrentamiento entre los poderes regio y nobiliario. ${ }^{17}$ Entre los miembros de la alta nobleza se habrían generado conflictos horizontales ${ }^{18}$ en una pugna constante por mejorar sus situaciones particulares con la concesión de riquezas, tierras y funciones gubernativas. A este efecto cabe señalar que tanto el "patrimonio" como la "privanza" (desempeño de papeles centrales en la administración regia) fueron dos criterios destacados en la subjetiva jerarquización de la nobleza en el siglo XIV. ${ }^{19}$

En 1308 tuvieron lugar las Vistas de Grijota entre Fernando IV y un grupo de nobles levantiscos, capitaneados por el infante don Juan y entre los que destacaba don Juan Manuel. El objetivo de la reunión fue forzar al Rey a que depusiese a sus oficiales para nombrar otros nuevos, más asiduos al grupo de los nobles rebeldes. Desde el punto de vista nobiliario, la consecución de dicho objetivo se habría visto como un gran triunfo, evidenciando una fuerza de grupo que el poder regio se veía obligado a tener en consideración. ${ }^{20}$

La estrecha vinculación de don Juan Manuel con el infante don Juan (obstinado y ambicioso capitán de la nobleza rebelde) en la persecución de unos objetivos más o menos comunes, sirvió para reforzar su idea de superioridad respecto al resto de la nobleza castellana en tanto que miembro de la familia real. En este sentido, destaca la forma en que ambos se enfrentaron juntos a un bando liderado por miembros de la alta nobleza como López de Haro y Núñez de Lara durante el desarrollo de lo que se ha estudiado como el pleito de Vizcaya y sus ulteriores consecuencias, que llevaron a don Juan Manuel y al infante don Juan a enfrentarse con el Rey. Aquí, sus reivindicaciones parecen haberse justificado, principalmente, en el trato por igual que Fernando IV dispensó a ambos bandos, sin tener en cuenta la mejoría que éstos últimos consideraban merecer dada su pertenencia a la familia real. A este efecto, uno de los principales motivos que impulsaron a don Juan Manuel a la rebeldía fue su insistencia al Rey para que se le entregase el cargo

17 En este sentido, Valdeón Baruque ya apuntó cómo los enfrentamientos de debieron a las tensiones entre sectores de un mismo grupo social (realeza-nobleza). Véase: Valdeón Baruque, J., Los conflictos sociales en el reino de Castilla en los siglos XIV y XV, Madrid, 19086, pp. 31-33.

18 Extrapolamos el concepto de su aplicación por Moxó, en similares condiciones, al caso de la competencia entre la nobleza urbana dentro de las aristocracias de las ciudades. Sobre esto véase: Moxó, S., "El auge de la nobleza urbana de Castilla y su proyección en el ámbito administrativo y rural a comienzos de la Baja Edad Media”, Boletín De La Real Academia De La Historia 178-3, 1981, pp. 486-488.

19 Moxó, S., "La nobleza", op. cit., pp. 89-94.

20 Sobre esto puede verse: González Mínguez, C., "Fernando IV de Castilla”, op. cit., pp. 330-331; González Mínguez, C., "Fernando IV de Castilla (1295-1312) perfil de un reinado”, p. 238. 
de mayordomo que éste le había prometido a cambio de su apoyo inicial en los enfrentamientos derivados del pleito de Vizcaya. ${ }^{21}$

\subsection{Consolidación de un estatus social durante la minoría de edad de Alfonso XI}

Tras la muerte de Fernando IV en el año 1312, los infantes don Juan y don Pedro encabezaron nuevos bandos nobiliarios opuestos que pugnaron por ganar el puesto de tutor del futuro Alfonso XI durante su minoría de edad. Don Juan Manuel, consolidado ya como uno de los señores más poderosos de Castilla, apoyó inicialmente a don Pedro ante la expectativa de obtener cierta participación en las funciones de gobierno, aunque más tarde se cambió al bando de don Juan. ${ }^{22}$ Cuando en el año 1315 se acordó que ambos infantes compartiesen la tutoría, la posición de don Juan Manuel se vio debilitada. No sólo había quedado finalmente apartado de las funciones de gobierno, sino que su cambio de bando provocó que don Pedro apoyase una revuelta de la población de Murcia que se negó a reconocerlo como adelantado. ${ }^{23}$

En este tiempo se confirmó el papel rector de los infantes en la política castellana sobre el resto de la nobleza, fortaleciéndose las consideraciones de don Juan Manuel a cerca de la superioridad social de la familia real. ${ }^{24}$ Ahora bien, la situación cambió en 1319 con la muerte de los tutores en una estrepitosa campaña militar en Granada. En tal situación, se volvieron a formar tres bandos enfrentados por la tutoría encabezados, respectivamente, por el propio don Juan Manuel, el infante don Felipe y don Juan el Tuerto, hijo del difunto infante don Juan. ${ }^{25}$

El rey Jaime II de Aragón, con cuya hija se había casado don Juan Manuel, lo instó a hacerse cargo de la situación aludiendo a la responsabilidad política que implicaban su poderío como señor y su altura social como miembro de la familia real castellana. ${ }^{26}$ Al mismo tiempo, la población de Murcia se replanteó su situación y volvió a aceptarlo como adelantado. ${ }^{27}$ Tras una serie de enfrentamientos iniciales entre los tres candidatos,

21 Sobre esto véase: Correa, M. P., "La integración de la nobleza en los oficios de la Corte de Fernando IV de Castilla (1295-1312)", Revista Chilena de Estudios Medievales, 9, 2016, p. 71; González Mínguez, C., "Fernando IV de Castilla (1295-1312) perfil de un reinado", pp. 237-239; González Mínguez, C., Fernando IV (1295-1312), op. cit., pp. 159-168, pp. 210-214, pp. 214-225 y pp. 227-231.

22 Sobre esto véase: Recuero Lista, A., "El reinado de Alfonso XI de Castilla (1312-1350)", Universidad Autónoma de Madrid, 2016, pp. 42-49; Giménez Soler, A., Don Juan Manuel, op. cit., pp. 51-53.

23 Sobre esto véase: Torres Fontes, J., "Murcia y don Juan Manuel”, op. cit., pp. 362-367; Giménez Soler, A., Don Juan Manuel, op. cit., pp. 53-62; Recuero Lista, A., "El reinado", op. cit., pp. 57-66 y pp. 301-304.

24 Sobre esto véase: García Fernández, M., "Alfonso XI. El Rey y su familia (1312-1350)”, en García Fernández, M. (coord.), El siglo XIV en primera persona: Alfonso XI, Rey de Castilla y León (13121350), Sevilla, 2015, pp. 21-24.

25 Sobre esto véase: Giménez Soler, A., Don Juan Manuel, op. cit., pp. 51-53; Sánchez-Arcilla Bernal, J., Alfonso XI 1312-1350, op. cit., pp. 92-94; Recuero Lista, A., "El reinado", op. cit., pp. 67-81.

26 A este efecto, véase la carta de don Jaime II a don Juan Manuel en el documento CCCXLVII de Giménez Soler: Giménez Soler, A., Don Juan Manuel, op. cit., pp. 478-479.

27 Sobre esto véase: Giménez Soler, A., Don Juan Manuel, op. cit., p. 91; Torres Fontes, J., "Murcia y don Juan Manuel", op. cit., p. 367; Recuero Lista, A., "El reinado", op. cit., pp. 301-303. 
en 1322 se estableció una nueva tutoría compartida, a pesar de lo cual continuaron las tensiones entre ellos. Don Juan Manuel se posicionó en contra del infante don Felipe, acercándose a don Juan el Tuerto, con el que compartía la misma posición social de hijo de infante. ${ }^{28}$ El periodo en que don Juan Manuel compitió por la tutoría y ejerció el cargo abarca desde 1320 a 1325 . Se trata del mismo periodo de tiempo en que compone la primera parte de su obra completa, la que se inserta en lo que hemos denominado como etapa alfonsí.

Consciente de la ambigüedad de su posición como hijo de infante, a lo largo del reinado de Fernando IV y la minoría de Alfonso XI don Juan Manuel vinculó su actividad a la de los infantes castellanos como rectores de la nobleza. Reforzó con ello su sentimiento de pertenencia a la familia real y su sentido de superioridad respecto a los demás nobles castellanos. Participó de forma activa tanto en las intrigas nobiliarias, como en funciones militares y en el desempeño de altos cargos como el de adelantado, mayordomo o tutor. Así, a pesar de ser un miembro menor de la familia real, consiguió situarse como uno de los personajes más destacados del panorama político y social castellano.

\section{EL GUSTO DE DON JUAN MANUEL POR LA CULTURA EN EL MARCO IDEOLÓGICO DEL MOLINISMO}

Para comprender la realidad de don Juan Manuel como escritor, así como la de su interés por la cultura y por la figura de su tío Alfonso X, es necesario atender, al menos, a dos cuestiones. En primer lugar, la inserción de don Juan Manuel en un contexto cultural específico que desde la historia de la literatura se ha estudiado bajo el apelativo de molinismo. Se trata de un marco ideológico originario de la corte de Sancho IV, que doña María de Molina mantuvo durante el tiempo de Fernando IV y se extendió hasta la minoría de Alfonso XI. En segundo lugar, deberíamos atender a la posibilidad de que su gusto por las letras se formase durante su infancia bajo la influencia de su madre doña Beatriz de Saboya primero, y del círculo de intelectuales de la sede arzobispal de Toledo después. En vista de todo ello, trataremos aquí dos puntos: 1) el molinismo como marco ideológico de don Juan Manuel durante el tiempo de Fernando IV; y 2) el gusto de don Juan Manuel por la cultura en el marco molinista.

\subsection{El molinismo como marco ideológico de don Juan Manuel durante el tiempo de Fernando IV}

Para comenzar la exposición sobre este punto, conviene realizar una explicación previa sobre el origen y connotaciones del concepto de molinismo, para así poder aclarar

28 Sobre esto véase: Giménez Soler, A., Don Juan Manuel, op. cit., pp. 66-77; A., "El reinado”, op. cit., pp. 69-71 y pp. 80-81; Sánchez-Arcilla Bernal, J., Alfonso XI 1312-1350, op. cit., pp. 97-99, pp. 104-112 y pp. $112-117$. 
la forma en que lo aplicaremos en este estudio. El concepto de molinismo fue acuñado por Fernando Gómez Redondo desde los estudios sobre la historia de la literatura y la cultura castellana medieval. ${ }^{29}$ Otros autores han seguido sus planteamientos, principalmente en los mismos campos de trabajo, aunque también se han dado casos de su aplicación en estudios de historia política. ${ }^{30}$ En su desarrollo original, se refiere al marco cultural forjado en la corte de Sancho IV y que, tras la muerte de este rey, fue extendido de mano de doña María de Molina desde la minoría de edad de su hijo Fernando IV hasta la de su nieto Alfonso XI. Se manifestó, principalmente, a través de una amplia producción literaria desarrollada en colaboración con el entorno intelectual del arzobispado de Toledo. Uno de los principales rasgos de esta producción es que nació como reacción al proyecto político-cultural de Alfonso X. ${ }^{31}$

Algunos de los valores que defendió el molinismo fueron la imposición de un profundo sentimiento de ortodoxia religiosa y la valorización de la figura del consejero regio como elemento esencial del gobierno. ${ }^{32}$ Para la prefiguración y transmisión eficaz de estos valores se adaptó el discurso narrativo a los gustos de la nobleza, dando lugar a un tipo de narrativa didáctica de ficción, marcadamente escenográfica, en la que se tratan, entre otros, temas de tipo político-social. ${ }^{33}$

La ortodoxia religiosa como uno de los valores fundamentales del molinismo se puede entender, en parte, como consecuencia del estrecho vínculo de la Casa Real con el círculo intelectual de la sede arzobispal de Toledo. Cabe destacar aquí la intensa labor cultural desarrollada por los arzobispos toledanos durante los siglos XIII y XIV. ${ }^{34} \mathrm{Re}-$

29 Gómez Redondo, F., Historia de la prosa medieval castellana, II, Madrid, 1999, pp. 1226-1291.

30 Para los estudios de tipo literario véanse: Rochwert-Zuili, Patricia, "El mecenazgo y patronazgo de María de Molina: pruebas e indicios de unos recursos propagandísticos y didácticos", e-Spania (2016), DOI: 10.4000/e-spania.25549; Funes, Leonardo, "Las letras castellanas en tiempos de Fernando IV: esbozo de una historia literaria", p. 534. Para el mismo uso del concepto desde la historia política véase: González Mínguez, C., "El perfil político de la reina María de Molina" Espacio, Tiempo Y Forma, 25, 2012, pp.239-254.

31 Sobre esto véase: Gómez Redondo, F., "El molinismo: un sistema de pensamiento letrado (1284-1350)", pp. 45-50; Gómez Redondo, F., "Doña María de Molina y el primer modelo cultural castellano”, pp. 29-30.

32 Para una vista general de los rasgos ideológicos del molinismo se pueden ver: Gómez Redondo, F., "El molinismo: un sistema de pensamiento letrado (1284-1350)", pp. 52-55; Gómez Redondo, F., "Doña María de Molina y el primer modelo cultural castellano", pp. 32-35; Gómez Redondo, F., "Don Juan Manuel", op. cit., pp. 828-830.

33 Para la idea de adaptación del discurso al público nos basamos aquí en las consideraciones de Van Dijk sobre lo que denominó como "creencias sobre las creencias mutuas" al estudiar los modelos contextuales del discurso ideológico. Véase: Van Dijk, Teun Adrianus, Ideología y discurso, pp. 36-40. Sobre el modelo literario propio de molinismo se pueden ver: Gómez Redondo, F., "Doña María de Molina y el primer modelo cultural castellano", pp. 35-45; Funes, Leonardo, "Las letras castellanas en tiempos de Fernando IV: esbozo de una historia literaria", pp. 358-359.

34 Se puede considerar que la base del estudio de estas relaciones entre la Corte de Sancho IV y la mitra toledana se encuentra en el trabajo de Orduna, cuyas consideraciones han sido seguidas por autores posteriores. Véase: Orduna, Germán, "La élite intelectual de la escuela catedralicia de Toledo y la literatura en época de Sancho IV", pp. 53-62; Gómez Redondo, F., "El molinismo: un sistema de pensamiento letrado (1284-1350)", p. 52-54; Bizzarri, Hugo Oscar, "Reflexiones sobre la empresa cultural del rey Don Sancho IV de Castilla": pp. 429-432. 
flejo de ello fue la importancia de su escuela catedralicia y la magnitud que adquirió su biblioteca capitular. ${ }^{35}$ Este carácter religioso dio pie a la generalización de una imagen altamente negativa de Alfonso X, caracterizando su preocupación intelectual como un rasgo distintivo de mal gobernante. ${ }^{36}$

Otro de los campos destacados de la producción alfonsí que se reelaboró en el marco cultural del molinismo fue el de la literatura "científica". Frente al enciclopedismo naturalista que caracterizó a los tratados del rey Sabio, las obras del entorno de Sancho IV y María de Molina se centraron en buscar la sencillez del contenido y en encuadrar en un marco religioso las temáticas alfonsíes.$^{37}$ En este sentido, se consideró que la vocación "científico-astrológica" de Alfonso X se distanciaba de la ortodoxia religiosa, contribuyendo a la construcción de una imagen negativa de su faceta intelectual.

En lo que respecta a la importancia de la figura del consejero, ${ }^{38}$ cabe apuntar que su puesta en valor ha de entenderse en relación con las elevadas cotas de poder que la alta nobleza y los infantes castellanos ganaron, especialmente, en la época de Fernando IV. Éste hizo del pactismo y la integración de la nobleza en las funciones administrativas y gubernativas de la Corona uno de sus principales instrumentos políticos. ${ }^{39}$ A menudo se buscó la solución de los conflictos nobiliarios a través de la conciliación, comprando la tranquilidad de los rebeldes y el servicio de los leales con cargos, tierras y rentas. En cierto modo, esta política no hizo más que continuar la que ya venía aplicando doña María de Molina desde la muerte de Sancho IV.40

Vemos así cómo la importancia del consejero se resaltó en el marco de un tipo de gobierno semicompartido entre el Rey y la nobleza que, eventualmente, resultó en una suerte de instrumentalización de la figura regia. ${ }^{41}$ De este modo, en el plano cultural se

35 Para la actividad intelectual del ámbito de la catedral de Toledo, independientemente de su vinculación con Sancho IV, así como para una visión general sobre la escuela catedralicia y la biblioteca capitular puede verse: Lop Otín, María José, "La catedral de Toledo, ente generador de cultural a fines de la Edad Media", pp. 357-374.

36 Sobre esto llamamos la atención sobre el trabajo de Leonardo Funes: Funes, Leonardo, "La blasfemia del rey Sabio: itinerario narrativo de una leyenda", Incipit, 3 (1993): pp. 51-70. Puede verse, también, el trabajo de Hugo Óscar Bizzarri: Bizzarri, Hugo Oscar y Rucquoi, Adeline, "Los espejos de príncipes en Castilla entre Oriente y Occidente", Cuadernos de historia de España 79, 2005, p. 16.

37 Gómez Redondo, F., "El molinismo: un sistema de pensamiento letrado (1284-1350)", p. 71.

38 Para la importancia de la figura del consejero regio en la clerecía cortesana molinista véase: Gómez Redondo, F., "El molinismo: un sistema de pensamiento letrado (1284-1350)", p. 54. De igual modo, llamamos la atención sobre el trabajo de Rochwert-Zuili en su estudio sobre El libro del caballero Zifar: Rochwert-Zuili, Patricia, "El valor del consejo en el Libro del caballero Zifar", e-Spania (2011). URL: http://journals.openedition.org/e-spania/20706.

39 Sobre esto véase: Moxó, S., "La nobleza", op. cit., pp. 94-99; Correa, Marcelo P, "La integración", op. cit., pp. 68-75; Valdeón Baruque, J, Los conflictos, op. cit., pp. 28-29; Santamaría Torquemada, Guillermo, "La legislación en cortes sobre la cancillería de Fernando IV: 1295-1312”: p. 299; González Mínguez, C., "El perfil político”, op. Cit., pp. 252-254.

40 González Mínguez, C., ““"El perfil político”, op. Cit., pp. 252-253.

41 La idea de una instrumentalización de la figura regia fue aplicada por Monsalvo para el caso de la nobleza trastamarista: Monsalvo, José María, "Historia de los poderes medievales del derecho a la antropología", p. 110. 
dio lugar a un tipo de "cortesía nobiliaria"42 que supone una de las formas más claras de reacción al proyecto cortesano de Alfonso X, que centraba la importancia y el poder en la persona del Rey. ${ }^{43}$

Sobre lo anteriormente expuesto podemos ver cómo la idea de un marco cultural molinista no se puede entender sin la existencia de unas pautas políticas que, al mismo tiempo, lo reproducen y lo moldean. A pesar de que el desarrollo del concepto de molinismo ha sido esencialmente literario-cultural, ya en su origen Gómez Redondo lo contempló con cierto cariz político. ${ }^{44}$ Se evidenciaba, con ello, la indisolubilidad de ambos aspectos, lo político y lo cultural, en el proyecto regio de doña María de Molina y Fernando IV. Ambos responden a una misma forma de pensar y comprender la política. Sobre esto podemos entender el molinismo como un tipo de marco ideológico, esto es, como un sistema de ideas y creencias generales compartido por uno o varios grupos de la misma sociedad. ${ }^{45}$

\subsection{El gusto de don Juan Manuel por la cultura}

Para comprender el gusto de don Juan Manuel por las letras, algunos autores han apuntado que se debería tener en cuenta la temprana influencia que su madre, doña Beatriz de Saboya, pudo haber tenido sobre él. ${ }^{46}$ Ésta se ocupó de su educación tras la muerte del infante don Manuel en 1283, hasta que ella falleció también en 1290. Si aceptamos como válido el testimonio que el autor presenta en su Libro de los estados cuando habla sobre la educación de los infantes, ${ }^{47}$ la formación recibida habría comenzado a partir de los cinco años, versando en diversas materias como la historia y el latín, así

42 Se puede ver un uso del concepto de "cortesía nobiliaria" como sinónimo sintético de molinismo por parte del propio Gómez Redondo en: Gómez Redondo, F., "Don Juan Manuel”, op. cit., p. 828. En la misma referencia se puede identificar también la puesta en valor del consejero como elemento esencial del molinismo.

43 Sobre esto pueden verse: Valdeón Baruque, Julio, "Cultura y política en tiempos de Alfonso X el Sabio", pp. 46-51; García de Cortázar, José Ángel, "El reinado de Alfonso X: un quicio entre dos épocas de la Edad Media", pp. 11-13; Rodríguez Llopis, M., "Alfonso X”, op. cit., pp. 107-117.

44 Véase esta doble consideración como "marco de relaciones políticas" y "ámbito de referencia literarias" en: Gómez Redondo, F., “Doña María de Molina y el primer modelo cultural castellano”, p. 30.

45 Van Dijk, Teun Adrianus, Ideología. Una aproximación multidisciplinaria, pp. 31-46.

46 Esta idea fue planteada en su momento por Manuel Alvar. Véase: Alvar, Manuel, "Alfonso X contemplado por don Juan Manuel”, Acatas del Congreso Internacional "La literatura en época de Sancho IV”, eds. Carlos Alvar y José Manuel Lucía Megías (Alcalá: Universidad de Alcalá, 1996), pp. 91-106.

47 Véase el capítulo XLVII del libro I en: Don Juan Manuel, El Libro de los Estados, eds. Robert Brian Tate e Ian R. Macpherson, pp. 148-150. Puede consultarse también: García Herrero, María del Carmen, "La educación de los nobles en la obra de don Juan Manuel", La familia en la edad media, coord. José Ignacio de la Iglesia (Nájera: Instituto de Estudios Riojanos, 2001), pp. 39-92; Pedraz, Miguel Vicente, "El imaginario corporal del Libro de los Estados. Representaciones somáticas de la sociedad y representaciones sociales del cuerpo en la obra política de Donjuán Manuel”, Studia histórica, 12 (1994): pp. 182-187. 
como en las cuestiones propias de un noble, como la equitación, la caza y el ejercicio de las armas. ${ }^{48}$

Parece posible que en algún punto tras la muerte de su madre don Juan Manuel se viese influenciado por las ideas de los intelectuales del círculo toledano. A este efecto, diferentes investigadores han destacado cómo el conjunto de la producción literaria de don Juan Manuel muestra notables afinidades con la producción cultural de Toledo. Aunque no tenemos constancia de la presencia de don Juan Manuel en la escuela catedralicia, sí que se han constatado las buenas relaciones entre éste y algunos personajes de la curia toledana. ${ }^{49}$ De este modo, es posible que su producción textual se desarrollase en un scriptorium propio, vinculado a su cancillería personal, ${ }^{50}$ pero bajo la influencia del ideario molinista toledano.

En este sentido, son varios los autores que han estudiado la localización de don Juan Manuel dentro del marco ideológico del molinismo atendiendo, principalmente, a su calidad de escritor de obras didácticas de contenido político. Entre los primeros estudios de este tipo destacó el de Richard P. Kinkade, que lo presentó como continuador de la narrativa didáctica de la corte de Sancho IV. ${ }^{51}$ Con ello, se pusieron de manifiesto algunas de las diferencias que lo separan de su tío Alfonso X en el aspecto literario. Más tarde resultarían decisivos los trabajos de Fernando Gómez Redondo dedicados, por un lado, al estudio de las líneas culturales e ideológicas de la prosa del molinismo ${ }^{52}$ y, por el otro, a la inserción del propio don Juan Manuel dentro de este marco cultural. A través de los planteamientos de Gómez Redondo parece haberse fijado la idea de que la raíz de la ideología de don Juan Manuel se hunde en el sustrato molinista, siendo uno de los autores que mejor recogieron su testigo.$^{53}$ En este punto llamamos la atención sobre el trabajo de Leonardo Funes, "Las letras castellanas en tiempos de Fernando IV: esbozo de una historia literaria”, a través del cual se puede notar la importancia de relacionar a don

48 Sobre esto véase: Giménez Soler, A., Don Juan Manuel, op. cit., pp. 1-3; Lacarra, M. J., Don Juan Manuel, op. cit., pp. 9-12; Flory, D., El Conde Lucanor, op. cit., , pp. 14-16; Rubio García, L., La minoridad, op. cit., p. 19-27.

49 Orduna, Germán, "La élite intelectual de la escuela catedralicia de Toledo y la literatura en época de Sancho IV”, pp. 60-61. Se puede consultar la también la relación que establece Hijano Villegas a raíz de los posibles orígenes de la Crónica Manuelina: Hijano Villegas, Manuel "Historia y poder simbólico en la obra de don Juan Manuel": pp. 77-78.

50 Sobre la existencia de scriptoria nobiliarios vinculados a las cancillerías de los señores en la época véase: Sanz Fuentes, María Josefa, "Cancillerías señoriales", pp. 326-32; Funes, Leonardo, "Las letras castellanas en tiempos de Fernando IV: esbozo de una historia literaria”, pp. 535-536.

51 Kinkade, Richard P., "Sancho IV: Puente literario entre Alfonso el Sabio y Juan Manuel", Modern Language Association 87, 5 (1972): pp. 1039-1051.

52 Gómez Redondo, F., "Doña María de Molina y el primer modelo cultural castellano”, pp. 29-46; "El molinismo: un sistema de pensamiento letrado (1284-1350)", pp. 45-81.

53 Gómez Redondo, F., "Don Juan Manuel”, op. cit., pp. 827-842. Se puede ver también, sobre la influencia posterior de don Juan Manuel como molinista en: Gómez Redondo, F., "Don Juan Manuel, Trastámara", Cahiers de linguistique et de civilisation hispaniques médiévales, 25 (2002): pp. 163-182. 
Juan Manuel con la producción cultural molinista propia del reinado de Fernando IV, y no tanto con la anterior de Sancho IV. ${ }^{54}$

Otros estudios más particulares sobre aspectos de la personalidad y la obra de don Juan Manuel han contribuido a reforzar estas consideraciones. Destacarían entre ellas, las afirmaciones sobre la profunda religiosidad del autor, ${ }^{55}$ las características marcadamente nobiliarias de sus planteamientos políticos y sociales, ${ }^{56} \mathrm{o}$ su inclinación hacia el desarrollo de las líneas didácticas. ${ }^{57}$

Si atendemos a su producción textual, podemos ver cómo la aproximación de don Juan Manuel a las líneas narrativas del molinismo se produce a partir de 1326 con una serie de obras que se pueden identificar como relatos de ficción didáctico-política. Entre ellas distinguimos El libro del caballero y el escudero, El libro de los estados y El libro enfenido. ${ }^{58}$ El tratamiento que se da a los temas políticos y sociales muestra una perspectiva marcadamente religiosa, reforzando el sentido didáctico con reflexiones teológicas

54 Funes, Leonardo, "Las letras castellanas en tiempos de Fernando IV: esbozo de una historia literaria", (ed.) Cesc Esteve, El texto infinito: tradición y reescritura en la Edad Media y el Renacimiento, Salamanca, Seminario de Estudios Medievales y Renacentistas-Sociedad de Estudios Medievales y Renacentistas, 2014, pp. 529-42.

55 Destacó, a este efecto, el trabajo de Rosa María Lida de Malkiel, que dio lugar a una fuerte consideración de la presencia del pensamiento dominico en don Juan Manuel; véase: Lida de Malkiel, Rosa María, “Tres notas sobre don Juan Manuel”, pp. 92-133. En la misma línea apuntó el trabajo de Inmaculada Urzainqui que relacionó los recursos didácticos de don Juan Manuel con esta orden religiosa; véase: Urzainqui, Inmaculada, "Más sobre la novedad didáctica de don Juan Manuel”: pp.701-728. También, siguiendo estos planteamientos, encontramos el trabajo de Francisco Rico: Rico, Francisco, "Don Juan Manuel and his connection with the Order of Preachers", Anuario de estudios medievales, 23 (1993): pp. 151-162.

56 Este tipo de consideraciones destacaron desde bastante temprano en el estudio de la representación social que hace el autor en El libro de los estados. Sobre ello pueden verse:

Ruíz, María Cecilia Literatura y política: el Libro de los estados y el Libro de las armas (Scripta Humanistica: Marylad, 1989); Stefano, Luciana de, "La sociedad estamental en las obras de don Juan Manuel, Nueva Revista de Filología Hispánica, 16 (1962): pp. 329-354; Maravall, José Antonio, "La sociedad estamental castellana y la obra de don Juan Manuel", Estudios de Historia del Pensamiento Español (Madrid: Cultura Hispánica, 1962), pp. 455-471.

57 Una consideración temprana sobre don Juan Manuel como autor didáctico se puede encontrar en la obra de Aldn Deyermond, sque lo destaca como autor en el florecimiento de este tipo de literatura en el XIV; véase: Deyermond, Alan, Edad Media. Historia de la Literatura Española, I, pp. 241-264. Más recientemente, el didactismo ha sido una de las líneas maestras que se pueden leer a lo largo de los monográficos que María Jesús Lacarra ha dedicado el autor; véase: Lacarra, M. J., Don Juan Manuel; Lacarra, M. J. (coord.), Don Juan Manuel y su producción literaria (Aula Medieval, 2014). URL: https:// parnaseo.uv.es/AulaMedieval/AulaMedieval.php?valor=autores\&lengua=es\#monografias; También Hugo Oscar Bizzarri y Adeline Rucquoi lo ha ubicado entre los productores de literatura didáctica molinista de corte nobiliario; véase: Bizzarri, Hugo Oscar y Rucquoi, Adeline, "Los espejos de príncipes en Castilla entre Oriente y Occidente", pp. 7-30. De igual modo, véase el trabajo personal de Bizzarri: Bizzarri, Hugo Oscar, "Las colecciones sapienciales castellanas en el proceso de reafirmación del poder monárquico (siglos XII y XIV)”, Cahiers de linguistique hispanique médiévale, 20 (1995): pp. 35-73.

58 Sobre esto véase: Kinkade, Richard P., "Sancho IV: Puente literario entre Alfonso el Sabio y Juan Manuel”, Modern Language Association 87, 5 (1972), p. 1049; Sturcken, H. Tracy, Don Juan Manuel, p. 105; Lacarra, M. J., Don Juan Manuel, op. cit., p. 36. 
y recursos discursivos propios de la predicación tales como el exemplum. ${ }^{59}$ Sobre esto se ha considerado que don Juan Manuel encontró en el didactismo una forma de autoexaltación como noble intelectual, considerando que aquel que enseña se posiciona por encima de aquel que aprende. De este modo, como afirmó Gómez Redondo, su escritura buscaría en gran medida "convertir en castigos el pensamiento nobiliario de que se sabe portador". ${ }^{60}$

La primera de estas obras, El libro del caballero y el escudero (1326-1327), resulta un ejemplo representativo de la forma en que don Juan Manuel reproduce el marco ideológico molinista al que pertenece. Cuando establece la situación inicial sobre la que arranca el relato, presenta a un rey ficticio caracterizado como modelo de buen gobernante, para el que la clave del éxito está en el mantenimiento de las buenas relaciones con la nobleza, con la que comparte el gobierno a través de la convocatoria frecuente de cortes. ${ }^{61}$ Luego, en la finalización de la primera parte de la trama, se incide, también, en que la buena formación de un noble es esencial para que éste pueda ser un buen consejero, a raíz de lo cual se pueden obtener cuantiosos beneficios del rey. ${ }^{62}$

\section{LA RECEPCIÓN DE ALFONSO X POR DON JUAN MANUEL DESDE EL MARCO IDEOLÓGICO DEL MOLINISMO}

A la hora de establecer etapas en la obra de don Juan Manuel, la crítica ha aceptado la existencia de una etapa alfonsí entre 1320 y 1325, coincidiendo con el periodo en que el autor fue tutor de Alfonso XI. Las obras aquí comprendidas se caracterizarían por ser resúmenes versionados de algunos de los escritos de Alfonso X.${ }^{63}$ Se incluirían, así, la

59 Sobre esto véase: Lida de Malkiel, Rosa María, "Tres notas sobre don Juan Manuel”, pp. 92-97; Urzainqui, Inmaculada, "Más sobre la novedad didáctica de don Juan Manuel”: pp. 701-710. De igual modo, Funes se sumó a las consideraciones sobre la influencia de los dominicos, planteando la posibilidad de que la materia teológica en la obre de don Juan Manuel fuese introducida por un monje de esta orden a través de un tipo de escritura delegada; véase: Funes, Leonardo, "Paradojas de la voluntad de autoría en la obra de don Juan Manuel", p. 132.

60 Gómez Redondo, F., "Don Juan Manuel”, op. cit., pp. 831-832.

61 Véasne los capítulos I y III del Libro del Caballero y el Escudero: Don Juan Manuel, Obras completas, Carlos Alvar y Sara Finci (eds.), Valencia, 2014, pp. 270-271.

62 Véase el capítulo XXIV: "El escudero fue muy bien recibido por el rey recompensado con riquezas y honra", en: Don Juan Manuel, Obras completas, Carlos Alvar y Sara Finci (eds.), op. cit., p. 279.

63 La crítica ha aceptado esta división a partir de su proposición por Germán Orduna en 1970. Este autor parece haberse basado, a su vez, en la división propuesta por Giménez Soler en 1932, que identificó las obras de la etapa didáctica como un núcleo diferenciado de textos dentro de la producción juanmanuelina. A este efecto véanse: Orduna, G., "Los prólogos", op. cit., p. 132 y p. 139. Para la aceptación de las etapas alfonsí y didáctica por parte de la crítica pueden verse: Leonardo Funes, "Don Juan Manuel y la herencia alfonsí", Actas del VIII Congreso de la Asociación Internacional de la Asociación Hispánica de Literatura Medieval, Santander, Asociación Hispánica de Cultura Medieval-Consejería de Cultura del Gobierno de Cantabria, 2000, p. 782; Lacarra, M. J., Don Juan Manuel, op. cit., pp. 21-22; Gómez Redondo, F., Historia de la Prosa Medieval Castellana, op. cit.p. 1102. 
Crónica abreviada, El libro de la caballería y El libro de la caza. Se podrían incluir también algunas obras perdidas de las que sólo tenemos constancia del título, como son El libro de las cantigas, El libro de las reglas como se debe trovar y El libro de los engeños. ${ }^{64}$ De igual modo, destaca la forma en que los prólogos de la Crónica abreviada y de El libro de la caza constituyen una exaltación explícita de Alfonso X como rey intelectual. ${ }^{65}$

En cierto modo se podría considerar que la reproducción de las obras alfonsíes por parte de don Juan Manuel responde a un fenómeno propio de la literatura del siglo XIV apuntado por Leonardo Funes, como fue el "agotamiento de las materias narrativas", que llevó a un retorno a la "textualidad previa bajo la forma de la continuación y del complemento". ${ }^{66}$ La crítica ha asumido la idea de que esta etapa inicial de su escritura (1320-1325) habría funcionado como un periodo de formación literaria, en la que don Juan Manuel habría adquirido autonomía como autor. ${ }^{67}$ Ahora bien, la lectura de las obras permite ver cómo el seguimiento de Alfonso X es más bien nominal, introduciendo variaciones de contenido y estilo ajenas al scriptorium alfonsí.

Parece que lo que más interesó a don Juan Manuel fue dibujar una figura específica de su tío el rey Sabio como modelo de conducta, como un tipo ideal de gobernante intelectual preocupado por la utilidad de la cultura como medio de engrandecimiento del reino. ${ }^{68}$ A través de este paralelismo, don Juan Manuel se habría apropiado de su autoridad intelectual, reforzando además su vínculo de pertenencia a la familia real en las disputas por la tutoría de Alfonso XI. ${ }^{69} \mathrm{Si}$ atendemos a los términos en que se elogia a Alfonso X en los prólogos de la Crónica Abreviada y de El libro de la caza, se puede apreciar cómo la imagen de intelectual o de rex sapiens funciona con una aplicación marcadamente política. En el primero, don Juan Manuel al ensalzar la faceta cultural de su tío se refiere a él con apelativos como el de "muy noble rey". ${ }^{70}$ Con ello, se presenta su inquietud

64 Sobre esto pueden verser: Leonardo, "Don Juan Manuel y la herencia alfonsî”: p. 783; Ayerbe-Chaux, Reinaldo, "El libro de los proverbios del conde Lucanor y de Patronio", Studies in Honour of Gustavo Correa, coord. Charles B. Faulhaber, Richard P. Kinkade, T. A. Perry (Maryland: Scripta Humanística, 1986), pp. 1-10; Ayerbe-Chaux, Reinaldo, "Las prosificaciones castellanas de las Cantigas de Santa María, ¿una obra perdida de don Juan Manuel?”, Bulletin of Cantigueiros, III (1990): pp. 39-52.

65 El tema ha sido tratado por Germán Orduna y más tarde, siguiendo sus postulados, por Leonardo Funes. Véase: Orduna, G., "Los prólogos", op. cit., pp. 123-144; Funes, L., "Don Juan Manuel y la herencia alfonsí”, Actas del VIII Congreso de la Asociación Internacional de Hispanista, Santander, 2000, pp. 781-788.

66 Funes, L., "Las letras castellanas en tiempos de Fernando IV: esbozo de una historia literaria", p. 533.

67 Lacarra, M. J., Don Juan Manuel, op. cit., p. 28; Funes, Leonardo, “Don Juan Manuel y la herencia alfonsî": pp. 782-788.

68 Sobre esto destacamos las conclusiones de Manuel Alvar sobre la utilidad de la labor cultural en el elogio de don Juan Manuel a Alfonso X. Véase: Alvar, Manuel, “Alfonso X contemplado por don Juan Manuel", pp. 103-105.

69 Sobre esto véase: Hijano Villegas, Manuel, "Historia y poder simbólico en la obra de don Juan Manuel": pp. 71-72; Funes, Leonardo, "Don Juan Manuel y la herencia alfonsî", p. 785; Saracino, Pablo Enrique, "La Crónica Abreviada de don Juan Manuel, una 'lectura desviada' de la Crónica Alfonsî”: pp. 7-8; Lacarra, M. J., Don Juan Manuel, op. cit., pp. 149-152.

70 Don Juan Manuel, Obras completas, Carlos Alvar y Sara Finci (eds.), op. cit., p. 46. 
intelectual como una forma de servicio a Dios y a la Corona. Presenta también la misma idea positiva de la producción cultural como servicio a Dios y al reino en el prólogo de $E l$ libro de la caza al afirmar que "ningún rey nin otro omne tanto fizesse por ello como él".71

\section{CONCLUSIÓN: LA EXALTACIÓN DEL GOBERNANTE INTELECTUAL COMO PROYECTO NEOALFONSÍ DE DON JUAN MANUEL}

Esta recepción positiva de la producción cultural alfonsí se produjo, como se ha indicado más arriba, en el entorno ideológicamente adverso del molinismo. Sobre ello, se puede notar que la elección de la figura de Alfonso X como modelo a seguir revierte una connotación política, que cobra sentido si atendemos al contexto histórico y biográfico de don Juan Manuel durante el tiempo de redacción.

Al presentarse como continuador de la labor cultural de Alfonso X, ${ }^{72}$ don Juan Manuel se exalta a sí mismo como gran conocedor de las materias básicas que conforman la educación de un noble, a saber: la historia como ámbito de enseñanza política, la ética y las características del estatus caballeresco, y la caza como actividad lúdico-formativa propia de la nobleza. De este modo, aplica sobre sí mismo la imagen de gobernante intelectual que había creado sobre el rey Sabio, lo que le permite, además, recalcar su parentesco con la familia real. ${ }^{73}$

Esta acción cobra especial sentido en el contexto de la participación de don Juan Manuel en las luchas por la tutoría de Alfonso XI entre 1320 y 1325. La auto-exaltación político-intelectual de don Juan Manuel y su vinculación con la familia real castellana a través de Alfonso X, le habrían permitido reforzar su candidatura como tutor frente a los otros aspirantes, el infante don Felipe y don Juan el Tuerto.

De este modo, la producción literaria que la crítica ha denominado como etapa alfonsí se pude entender como un proyecto neoalfonsí con una doble implicación, política y cultural. El objetivo de este proyecto habría sido el de robustecer los planteamientos de don Juan Manuel sobre su superioridad con respecto al resto de la nobleza castellana, insertándose con plena y total coherencia como un elemento más en el contexto políticobiográfico del autor.

71 Don Juan Manuel, Obras completas, Carlos Alvar y Sara Finci (eds.), op. cit., p. 213.

72 Para las referencias a la exaltación de la figura de Alfonso X en los prólogos véase: Orduna, G., "Los prólogos", op. cit., p. 132 y pp. 134-135.

73 Sobre esto véanse principalmente: Gómez Redondo, F., Historia de la Prosa Medieval Castellana, op. cit.p. 1102; Lacarra, M. J., Don Juan Manuel, op. cit., pp. 27-30. También: Funes, Leonardo, “Don Juan Manuel y la herencia alfonsî": p. 782; Saracino, Pablo Enrique, "La Crónica Abreviada de don Juan Manuel, una 'lectura desviada' de la Crónica Alfonsî’”: pp. 1-2. 


\section{BIBLIOGRAFÍA}

Alvar, Manuel, “Alfonso X contemplado por don Juan Manuel”, (eds.) Carlos Alvar y José Manuel Lucía Megías Acatas del Congreso Internacional "La literatura en época de Sancho IV”, Alcalá, Universidad de Alcalá, 1996, pp. 91-106.

Bizzarri, Hugo Oscar y Rucquoi, Adeline, "Los espejos de príncipes en Castilla entre Oriente y Occidente", Cuadernos de historia de España 79, 2005, pp. 7-30.

Bizzarri, Hugo Oscar, "Las colecciones sapienciales castellanas en el proceso de reafirmación del poder monárquico (siglos XII y XIV)", Cahiers de linguistique hispanique médiévale, 20, 1995, pp. 35-73.

Bizzarri, Hugo Oscar, "Reflexiones sobre la empresa cultural del rey Don Sancho IV de Castilla”, Anuario de estudios medievales 31, 1, 2001, pp. 429-451. https://doi. org/10.3989/aem.2001.v31.i1.287

Correa, Marcelo Paulo, "La integración de la nobleza en los oficios de la Corte de Fernando IV de Castilla (1295-1312)", Revista Chilena de Estudios Medievales, 9, 2016, pp. 63-77. https://doi.org/10.3989/aem.2005.v35.i2.149

Deyermond, Alan, Edad Media. Historia de la Literatura Española, I, Barcelona, Ariel, 1978.

Don Juan Manuel, El Libro de los Estados, eds. Robert Brian Tate e Ian R. Macpherson, Madrid, Castalia, 1991.

Don Juan Manuel, Obras completas, (eds.) Carlos Alvar y Sara Finci, Monografías, Aula Medieval, 3, 2014.

Flory, David, El Conde Lucanor: don Juan Manuel en su contexto histórico, Madrid, Pliegos, 1995.

Funes, Leonardo, "Don Juan Manuel y la herencia alfonsí", Actas del VIII Congreso de la Asociación Internacional de la Asociación Hispánica de Literatura Medieval, Santander, Asociación Hispánica de Cultura Medieval-Consejería de Cultura del Gobierno de Cantabria, 2000, pp. 781-788.

Funes, Leonardo, "La blasfemia del rey Sabio: itinerario narrativo de una leyenda", Incipit, 3, 1993, pp. 51-70. https://doi.org/10.4000/e-spania.25873

Funes, Leonardo, "Las letras castellanas en tiempos de Fernando IV: esbozo de una historia literaria", (ed.) Cesc Esteve, El texto infinito: tradición y reescritura en la Edad Media y el Renacimiento, Salamanca, Seminario de Estudios Medievales y Renacentistas-Sociedad de Estudios Medievales y Renacentistas, 2014, pp. 529-42.

Funes, Leonardo, "Paradojas de la voluntad de autoría en la obra de don Juan Manuel", (coords.) Florencio Sevilla Arroyo y Carlos Alvar Ezquerra, Actas del XIII Congreso de la Asociación Internacional de Hispanistas, Madrid, Editorial Castilla, I, 2000, pp. 126-133.

García de Cortázar, José Ángel, "El reinado de Alfonso X: un quicio entre dos épocas de la Edad Media”, (coord.) Miguel Rodríguez Llopis, Alfonso X y su época: el siglo del rey sabio, Barcelona, Carrogio, 2001, pp. 5-15. 
García Fernández, Manuel, “Alfonso XI. El Rey y su familia (1312-1350)”, (coord.) Manuel García Fernández, El siglo XIV en primera persona: Alfonso XI, Rey de Castilla y León (1312-1350), Sevilla, Universidad de Sevilla, 2015, pp. 19-35.

García Herrero, María del Carmen, "La educación de los nobles en la obra de don Juan Manuel”, (coord.) José Ignacio de la Iglesia, La familia en la Edad Media, Nájera, Instituto de Estudios Riojanos, 2001, pp. 39-92.

Giménez Soler, Andrés, Don Juan Manuel. Biografía y estudio crítico, Zaragoza, Academia, 1932.

Gómez Redondo, Fernando, "Don Juan Manuel, autor molinista”, en Margarita Freixas, Silvia Iriso Ariz y Laura Fernández (coords.), Actas del VIII Congreso Internacional de la Asociación Hispánica de Literatura Medieval, I, Santander, Asociación Hispánica de Cultura Medieval, 2000, pp. 827-842.

Gómez Redondo, Fernando, "Doña María de Molina y el primer modelo cultural castellano", El intercambio artístico entre los reinos hispanos y las cortes europeas en la Baja Edad Media, (coords.) María C. Cosmen, María Victoria Herráez Ortega, María Pellón Gómez-Calcerrada, León, Universidad de León, 2009, pp. 29-46.

Gómez Redondo, Fernando, "El molinismo: un sistema de pensamiento letrado (12841350)", Estudios de literatura medieval: 25 años de la Asociación Hispánica de Literatura Medieval, (coords.) Antonia Martínez Pérez y Ana Luisa Baquero Escudero, Murcia, Universidad de Murcia, 2012, pp. 45-81.

Gómez Redondo, Fernando, Historia de la Prosa Medieval Castellana, I y II, Madrid, 1998-1999.

González Mínguez, César, "El perfil político de la reina María de Molina” Espacio, Tiempo y Forma, 25, 2012, pp.239-254. https://doi.org/10.5944/etfiii.17.2004.3724

González Mínguez, César, "Fernando IV de Castilla (1295-1312) perfil de un reinado", Espacio, tiempo y forma, 17, 2004, pp. 223-244.

González Mínguez, César, "Fernando IV de Castilla (1295-1312): La guerra civil y el predominio de la nobleza", Universidad de Valladolid, 1976.

González Mínguez, César, Fernando IV (1295-1312), Palencia, La Olmeda, 1995.

H. Tracy Sturcken, Don Juan Manuel, Nueva York, Twayne, 1974.

Hijano Villegas, Manuel "Historia y poder simbólico en la obra de don Juan Manuel", Voz y Letrra, 25, 1-2, 2014, pp. 71-109.

Kinkade, Richard P., "Sancho IV: Puente literario entre Alfonso el Sabio y Juan Manuel”, Modern Language Association 87, 5, 1972, pp. 1039-1051. https://doi. org/10.2307/461181

Lacarra, María Jesús (coord.), Don Juan Manuel y su producción literaria, Monografías, Aula Medieval, 3, 2014.

Lacarra, María Jesús, Don Juan Manuel, Madrid, Síntesis, 2006.

Lida de Malkiel, Rosa María, "Tres notas sobre don Juan Manuel”, Estudios de literatura española y comparada, Buenos Aires, Eudeba, 1969, pp. 92-133. 
Lomax, Derek, "El padre de don Juan Manuel", Don Juan Manuel: VII Centenario, Murcia, Universidad de Murcia-Academia Alfonso X el Sabio, 1982, pp. 163-176.

Lop Otín, María José, "La catedral de Toledo, ente generador de cultural a fines de la Edad Media", Modelos culturales y normas sociales al final de la Edad Media, (coords.) Patrick Boucheron, Francisco Ruiz Gómez, Cuenca, Universidad de Castilla La Mancha_Casa de Velázquez, 2009, pp. 357-385.

López Serrano, Aniceto, Jaime II, don Juan Manuel y el Señorío de Villena, Villena, Instituto de Cultura "Juan Gil-Albert", 1999.

Maravall, José Antonio, "La sociedad estamental castellana y la obra de don Juan Manuel”, Estudios de Historia del Pensamiento Español, Madrid Cultura Hispánica, 1962, pp. 455-471.

Masià de Ros, Angels, "Las pretensiones de los infantes de la Cerda a la Corona de Castilla en tiempos de Sancho IV y Fernando IV: el apoyo aragonés”, Medievalia, 10, 1992, pp. 255-280.

Monsalvo, José María, "Historia de los poderes medievales del derecho a la antropología”, (coord.) Carlos Barros, Historia a debate: actas del Congreso Internacional "A historia a debate", 4, Santiago de Compostela, Historia a Debate, 1995, pp. 81-150.

Moreno Núñez, José Ignacio, “Algunas consideraciones y documentos sobre el régimen señorial en el tránsito de la Baja Edad Media”, Anuario De Estudios Medievales, 16, 1986, pp. 109-117.

Moxó, Salvador, "El auge de la nobleza urbana de Castilla y su proyección en el ámbito administrativo y rural a comienzos de la Baja Edad Media”, Boletín De La Real Academia De La Historia, 178, 3, 1981, pp. 407-518.

Moxó, Salvador, "La nobleza castellana en el siglo XIV”, (coords.) Pablo Sánchez León y Jesús Izquierdo, Clásicos de Historia Social de España: Una Selección Crítica, Valencia, Centro Francisco Tomás y Valiente (UNED), 2000, pp. 87-116.

Nussbaum, María Fernanda "La imagen jurídica del rey en la Crónica de Fernando IV", (coords.) Antonia Martínez Pérez y Ana Luisa Baquero Escudero, Estudios de literatura medieval: 25 años de la Asociación Hispánica de Literatura Medieval, Murcia, Universidad de Murcia, 2012, pp. 721-730.

O'Callaghan, Joseph F., "Las Cortes de Fernando IV: cuadernos inéditos de Valladolid 1300 y Burgos 1308”, Historia. Instituciones. Documentos, 13, 1986, pp. 315-328.

Orduna, Germán, "La élite intelectual de la escuela catedralicia de Toledo y la literatura en época de Sancho IV", Actas del Congreso Internacional "La literatura en época de Sancho IV”, (eds.) Carlos Alvar y José Manuel Lucía Megías, Alcalá, Universidad de Alcalá, 1996, pp. 53-62.

Orduna, Germán, "Los prólogos a la Crónica Abreviada y al Libro de la Caza: la tradición alfonsí y la primera época en la obra literaria de don Juan Manuel”, Cuadernos de Historia de España, 51-52, 1970, pp. 123-144. 
Ostolaza Elizondo, María Isabel, "La Cancillería y otros organismos de expedición de documentos durante el reinado de Alfonso XI (1312-1350)", Anuario De Estudios Medievales, 16, 1986, pp. 147-226.

Pedraz, Miguel Vicente, "El imaginario corporal del Libro de los Estados. Representaciones somáticas de la sociedad y representaciones sociales del cuerpo en la obra política de Donjuán Manuel”, Studia histórica, 12, 1994, pp. 182-187.

Recuero Lista, Alejandra, "El reinado de Alfonso XI de Castilla (1312-1350)", Universidad Autónoma de Madrid, 2016.

Rico, Francisco, "Don Juan Manuel and his connection with the Order of Preachers", Anuario de estudios medievales, 23, 1993, pp. 151-162.

Rochwert-Zuili, Patricia, "El mecenazgo y patronazgo de María de Molina: pruebas e indicios de unos recursos propagandísticos y didácticos", e-Spania, 2016, DOI: 10.4000/e-spania.25549.

Rochwert-Zuili, Patricia, "El valor del consejo en el Libro del caballero Zifar", eSpania, 2011. URL: http://journals.openedition.org/e-spania/20706. https://doi. org/10.4000/e-spania.20706

Rodríguez Llopis, Miguel, “Alfonso X, rey de Castilla y León (1252-128), (coord.) Miguel Rodríguez Llopis, Alfonso X y su época: el siglo del rey sabio, Barcelona, Carrogio, 2001, pp. 107-125.

Rubio García, L., La minoridad de don Juan Manuel y la ocupación aragonesa de Murcia (1282-1296), Murcia, Real Academia Alfonso X el Sabio, 2000.

Ruíz, María Cecilia, Literatura y política: el Libro de los estados y el Libro de las armas, Marylad, Scripta Humanistica, 1989.

Sánchez-Arcilla Bernal, Jánchez, Alfonso XI 1312-1350, Palencia, Diputación Provincial, 1995.

Santamaría Torquemada, Guillermo, "La legislación en cortes sobre la cancillería de Fernando IV: 1295-1312”, Las Cortes de castilla Y León, 1188-1988: Actas de la tercera etapa del Congreso científico sobre la historia de las Cortes de Castilla y León, I, Valladolid, Simancas-Cortes de Castilla y León, 1988, pp. 285-300.

Sanz Fuentes, María Josefa, "Cancillerías señoriales", (dir.) Juan Ignacio Ruiz de la Peña Solar, La nobleza peninsular en la Edad Media. VI Congreso de Estudios Medievales, Avila, Fundación Sánchez Albornoz, 1999, pp. 326-334.

Saracino, Pablo Enrique, "La Crónica Abreviada de don Juan Manuel, una 'lectura desviada' de la Crónica Alfonsí”, Medievalia, 8, 2006, pp. 1-10.

Stefano, Luciana de, "La sociedad estamental en las obras de don Juan Manuel", Nueva Revista de Filología Hispánica, 16, 1962, pp. 329-354. https://doi.org/10.24201/ nrfh.v16i3/4.1444

Torres Fontes, Juan, "Murcia y don Juan Manuel: tensiones y conflictos", Don Juan Manuel: VII centenario, Murcia, Universidad de Murcia-Academia Alfonso X el Sabio, 1982, pp. 353-383. 
Urzainqui, Inmaculada, "Más sobre la novedad didáctica de don Juan Manuel”, Bulletin Hispanique, 92, 2, 1990, pp. 701-728. doi: https://doi.org/10.3406/hispa.1990.4718 Valdeón Baruque, Julio, "Cultura y política en tiempos de Alfonso X el Sabio", (coords.) Patrick Boucheron, Francisco Ruiz Gómez Modelos culturales y normas sociales al final de la Edad Media, Cuenca, Universidad de Castilla La Mancha-Casa de Velázquez, 2009, pp. 39-52.

Valdeón Baruque, Julio, Los conflictos sociales en el reino de Castilla en ellos siglos XIV y XV, Madrid, Siglo Veintiuno, 1986.

Van Dijk, Teun Adrianus, Ideología y discurso, Barcelona, Ariel, 2003.

Van Dijk, Teun Adrianus, Ideología. Una aproximación multidisciplinaria, Barcelona, Gedisa, 1999. 